\title{
Biological liquid crystal elastomers
}

\author{
David P. Knight ${ }^{1^{*}}$ and Fritz Vollrath ${ }^{1,2}$ \\ ${ }^{1}$ Zoology Department, University of Oxford, South Parks Road, Oxford OX1 3PS, UK \\ ${ }^{2}$ Zoology Department, Universitetsparken B135, 8000 Aarhus C, Denmark
}

\begin{abstract}
Liquid crystal elastomers (LCEs) have recently been described as a new class of matter. Here we review the evidence for the novel conclusion that the fibrillar collagens and the dragline silks of orb web spiders belong to this remarkable class of materials. Unlike conventional rubbers, LCEs are ordered, rather than disordered, at rest. The identification of these biopolymers as LCEs may have a predictive value. It may explain how collagens and spider dragline silks are assembled. It may provide a detailed explanation for their mechanical properties, accounting for the variation between different members of the collagen family and between the draglines in different spider species. It may provide a basis for the design of biomimetic collagen and dragline silk analogues by genetic engineering, peptide- or classical polymer synthesis. Biological LCEs may exhibit a range of exotic properties already identified in other members of this remarkable class of materials. In this paper, the possibility that other transversely banded fibrillar proteins are also LCEs is discussed.
\end{abstract}

Keywords: self-assembly; fibril; mesogen; nanofibril; amphiphilic; block copolymer

\section{INTRODUCTION}

Liquid crystals have been defined as a remarkable, paradoxical state of matter intermediate between true liquids and true crystals because their molecules, or regions within them, have a mobility similar to that of true liquids yet they show an orientational and sometimes positional order similar to that of solid crystals (Collings 1990). While most types of liquid crystal are able to flow, LCEs, also described as solid liquid crystals, are not. It is only recently that LCEs have been proposed as being a novel state of matter (Warner \& Terentjev 1996), although the theoretical existence of this state was first postulated more than 25 years ago by de Gennes (1969, 1975). The term 'solid liquid crystal' was originally used in a now outmoded way to describe the more heavily cross-linked nematic resins (Bouligand et al. 1974). However, it was not until the late 1980s and early 1990s that LCEs were first synthesized by Finkelman's group at Freiburg University (Barclay \& Ober 1993; Schneider et al. 2000).

Both classical rubbers and LCEs are weakly cross-linked polymers showing reversible extensibility and energy storage (Legge et al. 1991). A major difference between these two classes of materials is that the polymer chains in classical rubbers are highly disordered at rest while those of LCEs show orientational order, earning them the term 'anisotropic elastomers' (Texeira 1999). LCEs can be defined as lightly cross-linked main-chain or side-chain (Northolt \& Sikkema 1991) liquid crystalline polymers showing elastomeric properties. Their mesogenic (liquidcrystal forming) segments can exist in a number of different liquid crystalline phases (mesophases) including nematic, smectic, twisted nematic (cholesteric) (Meier \&

*Author for correspondence (knight@tegdown.u-net.com).
Finkelmann 1993), hexagonal (Schneider et al. 2000) and lamellar (Fischer et al. 1995). These mesophases represent different energy minima that depend on different balances between weak attractive and repulsive forces acting on the mesogenic units (de Gennes 1974; Collings 1990). Mesogenic groups must be chiral (displaying a singlehandedness), relatively stiff and rod-shaped or prolate- or oblate spheroids (de Gennes 1974). Lamellar liquid crystal elastomers (LLCEs) show an alternation of hydrophobic and hydrophilic segments in alternating layers (lamellae) and are formed from amphiphilic AB block copolymers (Fischer et al. 1995; King et al. 1999; Fraaije et al. 2000; Hamley 2000). The formation of lamellae is driven by the segregation of the block copolymer's hydrophobic A blocks from the more hydrophilic B blocks. Amphiphilic AB block copolymers also show a remarkable ability to self-assemble into nanofibrils and other hierarchical textures (Chan et al. 1992; Putthanarat et al. 2000; Qu et al. 2000).

The mesogenic units in LCEs, as in other liquid crystals, show a statistically defined orientation distribution about a direction known as the liquid crystal director (de Gennes 1974). The director field is coherent throughout a volume known as a domain. Monodomain liquid crystal elastomers are constructed from a single domain and are more common and better understood than the polydomain ones (with many domains, each possessing their own director field). Cross-links at nodes hold together the polymer chains of LCEs into a solid meshwork, but the mesogenic units retain at least some degree of liquid-like mobility (Texeira 1999). This mobility depends on three factors: the flexiblility of spacer links (Mitchell et al. 1992) that join the mesogenic segments to the cross-linked nodes of the meshwork; the relatively small number of crosslinked regions in the network (Warner \& Terentjev 1996); and, as in other liquid crystals (Collings 1990), on weak 
repulsive forces balancing the attractive ones between the mesogenic units. The mesogenic domains are free to change their orientation and position relative to one another (Mao et al. 1998).

The elasticity of LCEs is thought to depend on a mechanical coupling through cross-links between the applied strain and the orientation of the mesogenic units (Legge et al. 1991), though the detailed workings of this coupling are still far from clear (Uchida 2000). When a deforming force is applied to the mesogenic units there is usually a change in their statistical orientation relative to the original director and a consequential change in shape of the polymer molecule as a whole, accompanied by an increase in free energy. The increase in free energy can be used to restore the chains to their preferred, energy-minimized state. This means that the classical Gaussian theory of rubber elasticity (Treloar 1975) can be applied, with suitable extension, to LCEs (Mao et al. 1998; Warner et al. 1988). Under certain circumstances, however, the whole distribution of the mesogenic units (director) can be rotated in such a way that the shape of the molecules continues to reflect the shape of the sample of material as a whole. This rotation therefore occurs without distortion of the polymer chains and consequently without a change in free energy, giving them what is known as soft elasticity, one of several exotic properties found in LCEs. Soft elasticity results in wholly or partly flattened regions in the material's stress-strain curve that are not seen in isotropic rubbers (Texeira \& Warner 1999; Uchida 2000).

Monodomain LCEs can be prepared in two ways: (i) by cross-linking a liquid crystalline monomer in its liquid crystalline state, and (ii) by lightly cross-linking a liquid crystalline monomer in its isotropic (disordered state), applying sufficient mechanical stress to order the molecules and then cross-linking them again (Warner \& Terentjev 1996).

Having described LCEs from a perspective of material science, we aim to show that fibrils of transversely banded collagens and the nanofibrils of spider dragline silks can be regarded as LLCEs. To do this it is necessary to satisfy the following criteria taken from the above description of the structure, formation and properties of LCEs. (i) The fibrils must have the structure of an LLCE. That is, they must be lightly cross-linked networks in which some segments exhibit a liquid-like mobility; and there is an alternation of hydrophobic and hydrophilic lamellae. (ii) The initial assembly of the fibrils is from mesogenic, amphiphilic AB block copolymeric 'monomers', is thermodynamically reversible and depends on the existence of the balance between weak attractive and repulsive interactions such as hydrophobic/hydrophilic effects and coulombic forces. (iii) The fibrils show reversible extensibility.

We then consider the predictive value of classifying collagens and spider dragline silks as LLCEs. Finally, we suggest that other amphiphilic block copolymeric fibrous proteins forming transversely banded fibrils may be LLCEs.

\section{EVIDENCE THAT FIBRILLAR COLLAGENS ARE LLCES}

(a) Liquid crystalline structure

The first evidence for the liquid crystallinity of a fibrillar collagen with a classical $68 \mathrm{~nm}$ axial banding pattern came from the study of elastoidin, which has tapered giant fibrils up to $2 \mathrm{~mm}$ in diameter. These give resilience to the fins of cartilaginous fishes (Woodhead-Galloway \& Knight 1977) and the term 'elastoidin' derives from their rubber-like properties when wet, completely and rapidly straightening after being bent double. Elastoidin shrinkage temperature and insolubility are both high (Woodhead-Galloway \& Knight 1977), indicating that the molecules are crosslinked into a network, a necessary prerequisite for an LCE. The low-angle X-ray scattering of wet elastoidin showed only diffuse scattering on the equator indicating that the transverse arrangement of the molecules was completely liquid-like despite the fact that the highly ordered longitudinally staggered arrangement of molecules is closely similar to that of conventional mammalian collagen fibrils (Woodhead-Galloway \& Knight 1977) found, for example, in tendons.

Findings on elastoidin led to a re-examination of the low-angle equatorial X-ray diffraction pattern of rat-tail tendon fibrils. These demonstrated a combination of sharp Bragg reflections and diffuse scatter suggesting that the side-to-side arrangement in some regions of the axially repetitive structure of the fibril were crystalline, while others were liquid-like (Woodhead-Galloway \& Knight 1977). A comparison of the X-ray diffraction of wet and dry elastoidin gave further evidence for liquid crystallinity, as follows. The molecules were axially orientated when wet but tilted sharply on drying and a similar tilting occurs in other collagen fibrils (Hukins et al. 1976), as would be expected in a smectic A to smectic C liquid crystal phase transition. Taken together, these observations led to the suggestion that elastoidin and other transversely banded collagen fibrils were liquid crystalline (Hukins \& Woodhead-Galloway 1977, 1978). The diffuse equatorial scattering of elastoidin has recently been reinvestigated and shown to involve two components, one of which is Gaussian. This component changed as the water content was altered (Lees 1998) and is thought to result from side-toside movements of collagen segments produced by thermal agitation, suggesting the mobility required for liquid crystallinity. In rat-tail tendon fibrils, a high proportion $(80 \%)$ of the equatorial scattering comes from liquid-like segments, while only $20 \%$ comes from ordered segments (Hulmes et al. 1995), as might be expected in an LCE.

Further support for the liquid crystallinity of collagen fibrils came from improved models for rat-tail tendon collagen fibrils. A crimped model in which highly mobile molecular segments of collagen in the gap regions are linked by highly ordered straight-tilted segments in the overlap region explained the sharp reflections and diffuse scatter of the low-angle equatorial X-ray diffraction pattern (Parry 1988). The gap region has a much higher water content, lower packing fraction and lower content of imino and aromatic amino acids thought to contribute to the rigidity of the collagen helix (Fraser et al. 1987). Thus the evidence from X-ray diffraction, together with evidence for formation of networks by systematic rather than random intermolecular covalent cross-linking (Fraser 1998; Knott \& Bailey 1998), suggest a strong resemblance between collagen fibrils and LCEs.

The mobility of different transverse zones of the conventional collagen fibril is not fully understood. The $4: 1$ ratio of mobile to immobile segments (Hulmes et al. 1995) 


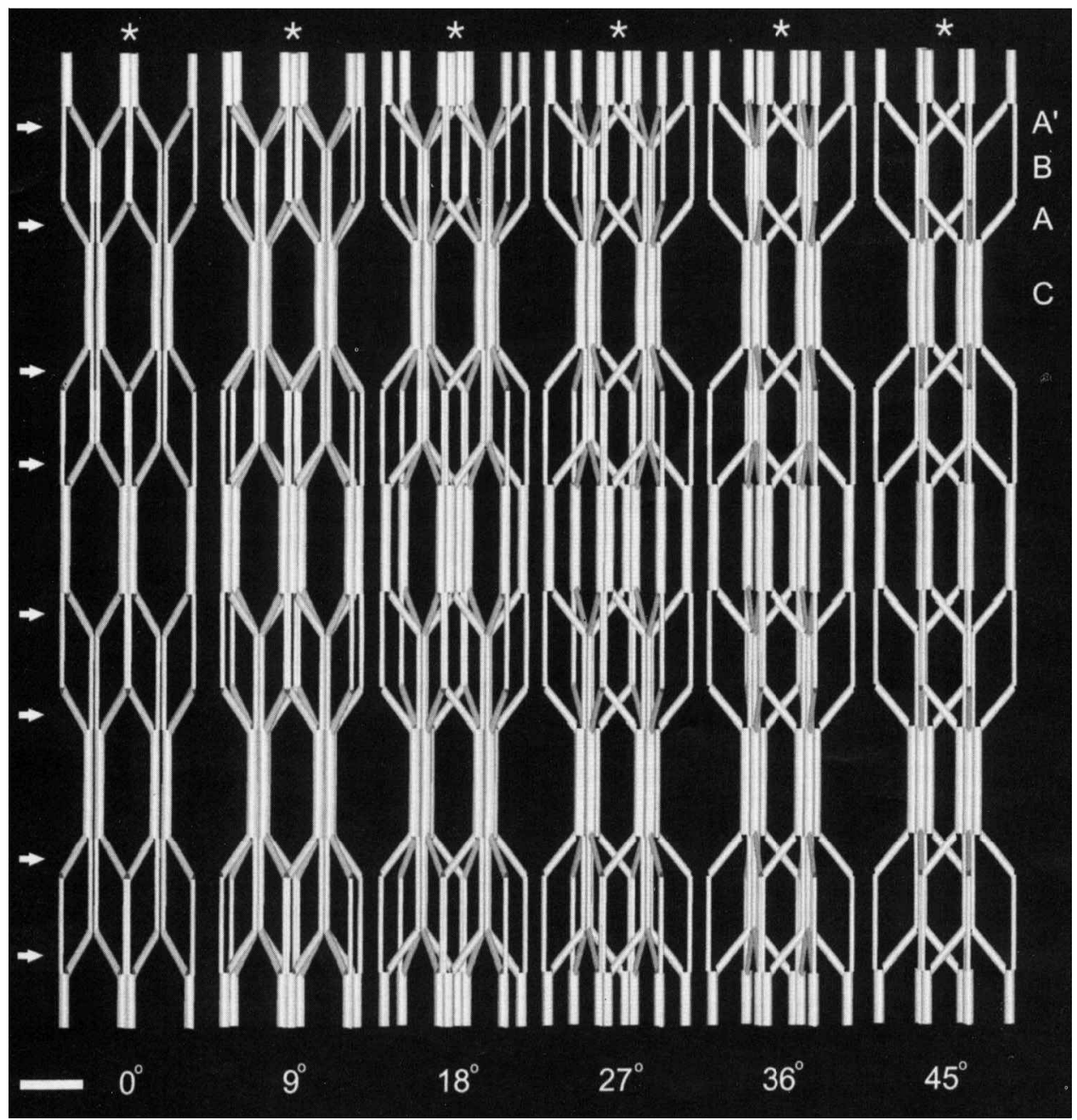

Figure 1. A stereo gallery showing four unit cells to illustrate the open network molecular construction of dogfish EC collagen fibrils from Knupp et al. (1998). The structural similarity to an LLCE is immediately obvious. The figure can be viewed as a series of stereo images of the unit cell at different rotations. The arrows point to the A or $\mathrm{A}^{\prime}$ bands. The axial repeat period is $40 \mathrm{~nm}$.

suggests that the mobile segments are not limited solely to the gap region. Evidence from molecular modelling suggests that the regions of the collagen molecule rich in hydrophilic residues are less perfectly triple helical than those rich in hydrophobic amino acids (Brown et al. 1997) and this may contribute to differences in flexibility. In addition, we have suggested that an anomalous region of the rat-tail tendon fibril may be particularly mobile and serve as a dashpot close to the principal intermolecular cross-link (Hu et al. 1997).

In addition to the work on conventionally banded collagen fibrils, there is evidence that the unconventionally banded fibrils of dogfish EC collagen also have the structure of an LCE. These fibrils show an axial periodicity of $40 \mathrm{~nm}$ and are formed from a much shorter collagen molecule than that of rat-tail tendon collagen (Knight et al. 1996). Like elastoidin, dogfish EC collagen is highly insoluble and has a very high thermal shrinkage temperature indicating the existence of a cross-linked meshwork. Figure 1 shows the regularly kinked, meshwork structure of these fibrils (Knupp et al. 1998; Knupp \& Squire 1998). The meshwork is thought to be held together, in part, by covalent bonds between the ends of the molecules (Knight \& Vollrath 2001a) that are thought to lie in nar- row bands that strongly exclude negative stain (Knight $\&$ Hunt 1974). The fibrils have been described as a crosslinked lamellar liquid crystal (Knight et al. 1993). The structure of assembly states in other unconventional collagens, such as the hexagonal columns seen in type VIII collagen (Ricard-Blum et al. 2000), suggests that many other collagens could be liquid crystalline and represent other LC phases.

The hydrophobic residues of collagens tend to be grouped into several transverse bands within the axial period of the fibril (Chapman et al. 1990; Gathercole et al. 1993; Ortolani et al. 1994) as in LLCEs.

\section{(b) Liquid crystalline assembly}

Martin et al. (2000) have shown that type I procollagen, the precursor for mammalian type I collagen fibrils, forms nematic and pre-cholesteric liquid crystals in vitro and have argued that this accounts for the assembly of crimped fibrils and other assemblies in vivo. We have also presented evidence from chemically fixed material that dogfish EC collagen fibrils are assembled from a liquid crystalline precursor that undergoes a number of liquid crystal phase transitions during storage and secretion (Knight et al. 1993). The length-to-width ratio (ca. 20) 
of dogfish EC collagen molecules is highly favourable for nematic liquid crystallization (Knight et al. 1996).

Sequence data obtained for many other members of the collagen family clearly demonstrate that they are block copolymers with an amphiphilic nature arising from the tendency of hydrophobic and hydrophilic amino acids to be segregated into separate regions in the molecule (Ricard-Blum et al. 2000; Knupp \& Squire 2001). This molecular structure is a prerequisite for the formation of LLCEs.

Weak forces, including hydrophobic, hydrophilic and coulombic interactions, are important in establishing the stagger that gives rise to the axially banded structure of fibrillar collagens (Hulmes et al. 1973; Wallace 1985; Leikin et al. 1995), as would be expected for lyotropic liquid crystals (Collings 1990). An analysis of the assembly of highly ordered sheets of type VI collagen, closely analogous to those of dogfish EC collagen, clearly demonstrates that type VI collagen is an amphiphilic AB block copolymer and that hydrophobic and coulombic interactions are important in its assembly (Knupp \& Squire 2001).

The earliest stages in the assembly of type I collagen fibrils are thermodynamically reversible, as would be expected for a liquid crystal phase transition (see brief review in Knight et al. (1993)).

\section{(c) Elastomeric properties}

Thus, the structure of transversely banded collagens, the mobility of some of their molecular segments and their method of assembly strongly suggest that they are LLCEs, but do they show elastomeric properties? A short answer to this question is complicated by the fact that tissues or structures containing fibrillar collagen are, by definition, fibrous composites. This means that the bulk mechanical properties depend, at least to some degree, on the interfibrillar matrix and the arrangement of fibrils within the material. Inferences about the mechanical properties of the fibrils themselves cannot, therefore, be derived directly from the bulk properties. However, the practically uniaxial orientation of collagen fibrils in slightly stretched tendons and the small concentration of matrix in this tissue simplifies the problem, enabling the conclusion that tendon collagen fibrils must be quite stiff (Vincent 1982). Subtler approaches are required to examine the tensile properties of the fibrils directly.

The effect of strain on conventional collagen fibrils has been investigated by low-angle synchrotron X-ray diffraction (Misof et al. 1997). This demonstrated a completely reversible extension of the axial period during cyclical loading in the 'toe in' region of the stress-strain curve and an increase in lateral molecular order accompanied by a reduction of mobility with strain. The extension of the axial period is thought to arise from the straightening out of thermally activated molecular kinks. Such tensile behaviour is compatible with that of an LLCE.

A reversible extension of the axial period has also been demonstrated in dogfish EC collagen (Hepworth et al. 1994). This material is much tougher than tendon and can be extended by up to $39 \%$ before rupture. Low-angle $\mathrm{X}$-ray diffraction and TEM indicated that the axial period increased reversibly by $8 \%$ when the material was subjected to a global strain of $24 \%$. As in conventional collagen fibrils, this reversible extension could be accounted for by a straightening out of the molecular kinks.

The mobility and rigidity of the collagen molecules under progressive strain have also been investigated by solid-state proton NMR in mammalian arterial wall after the selective removal of elastin. This approach indicated that a reduction in mobility of the chains accounts for the slope of the stress-strain curve of the bulk material at high strain (Vinee et al. 1993), as would be expected in an elastomer. These findings suggested a model for the elasticity of collagen closely similar to that proposed by Misof et al. (1997) (see above).

Finally, the effect of temperature on increasing the stiffness of collagen fibrils in suspension has been successfully modelled using the assumption that mechanically, the fibrils behave like lyotropic liquid crystal polymers (Rosenblatt et al. 1993).

\section{EVIDENCE THAT ORB WEB SPIDER DRAGLINE SILKS ARE LLCEs}

\section{(a) Liquid crystalline structure}

There is a consensus that the dragline silk of orb web spiders contains $\beta$-crystallites largely composed from the polyalanine segments (Jelinski 1998; Riekel et al. 1999) separated by the more mobile and less ordered glycinerich segments (Gosline et al. 1984, 1994; Termonia 1994). X-ray diffraction indicated that the $\beta$-crystallites were approximately axially orientated with respect to the fibre (see reviews and further evidence in Grubb \& Ji (1999); Riekel et al. (1999)). These observations supported a model (Termonia 1994) for silk as a classical entropic rubber in which isolated $\beta$-crystallites with an axial orientation are set in a tangle of disordered segments. It is, however, unclear what orientated the isolated crystallites and Termonia's model did not fit all the experimental data (Termonia 1994; Kümmerlen et al. 1996; Simmons et al. 1996).

Evidence has accumulated for an improved model for spider dragline silk in which the major component is an orientated nanofibril composed of transverse lamellae that show a regular alternation of axially orientated $\beta$-crystallites and fewer crystalline glycine-rich domains (figure 2). Four lines of evidence strongly support such a model. (i) ${ }^{13} \mathrm{C}$ Two-dimensional NMR spin-diffusion measurements on Nephila dragline silk indicated that both polyalanine $\beta$-crystallites and 3(1) helices derived from the glycinerich segments are orientated fairly precisely in line with the thread (Kümmerlen et al. 1996; Van Beek et al. 1999). (ii) A study of amide I shifts obtained by Raman microscopy from single spider dragline threads (Sirichaisit et al. 1999; Yeh \& Young 1999) showed that both $\beta$-crystallites and glycine-rich segments experience uniform stress during extension of the thread. This strongly suggests that both components lie within lamellar micro- or nanofibrils in the thread. (iii) TEM (see figures 3 and 4), SEM, small-angle X-ray diffraction and AFM gave strong evidence for the existence of nanofibrils with a diameter of $c a .5 \mathrm{~nm}$ in spider dragline silk (for review see Putthanarat et al. (2000)). Furthermore, nanofibrils of this diameter can be assembled from dilute aqueous solutions of spider silk dope (Chen et al. 2001) or a polypeptide multiblock 


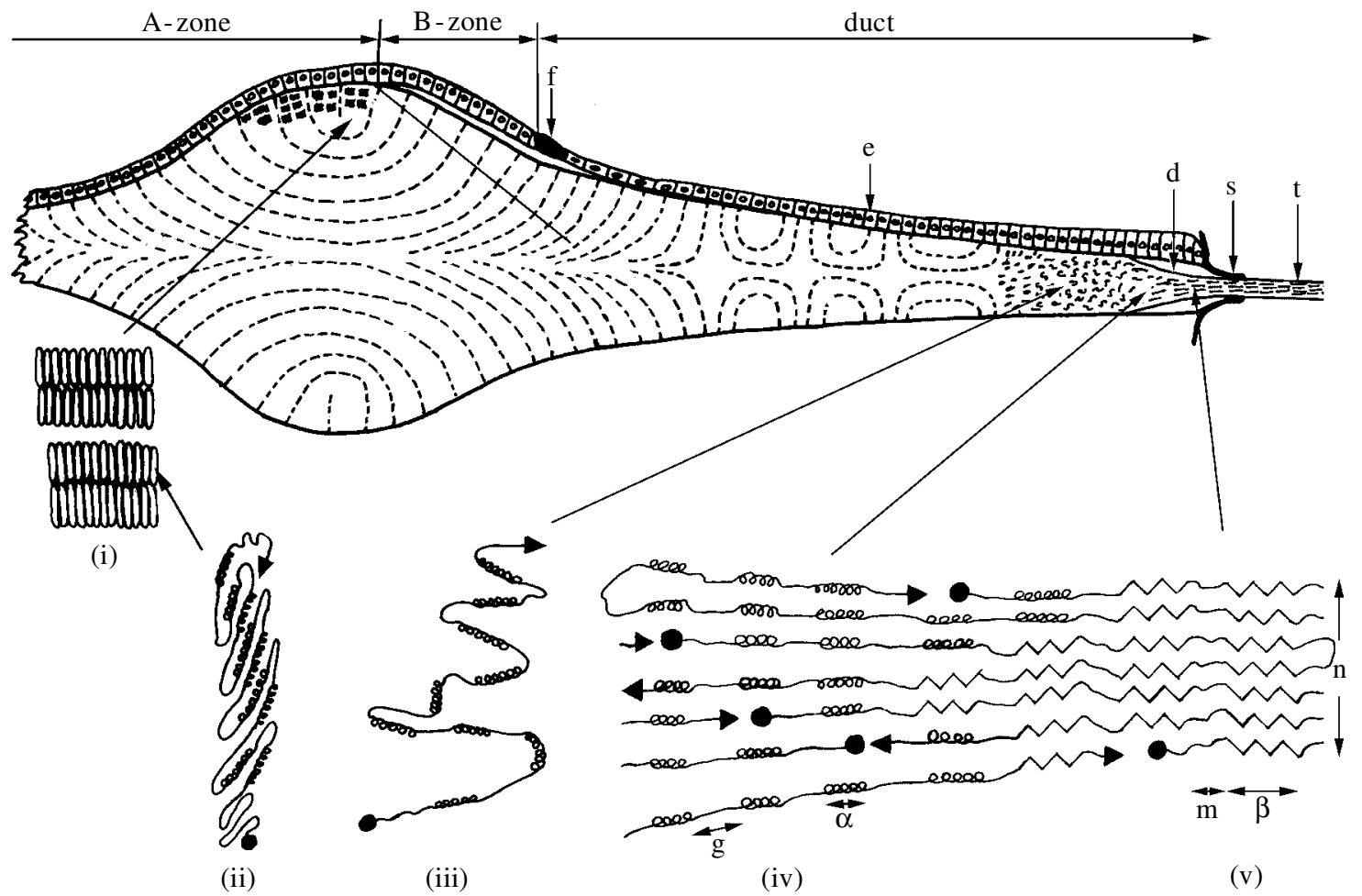

Figure 2. The proposed stages in lamellar liquid crystalline assembly of the nanofibrils of the MA (dragline) silk thread in orb web spiders (see text) showing: (i) a diagrammatic optical section of the gland and duct (upper part of illustration); nematic discotic units; (ii) rod-shaped molecules of spidroin; (iii) partly unwound molecules; (iv) early stage of formation of the solid fibre and ( $\mathrm{v}$ ) fully formed fibre (see text). The lumen of the gland has been represented as much wider in proportion to length with only a small number of bi-layer discs (top left) and the epithelium, (e), only on one side of the duct and gland. The dotted lines represent the molecular director field. This lies at right angles to the slow axis of polarization as a result of the assembly of the compactly wound, rod-shaped molecules of spidroin (ii) into bi-layered discs of the nematic discotic phase (i). These are present as an escaped nematic texture in the gland proper and first half of the duct (upper half of figure). Funnel (f), draw down taper (d), spigot (s), thread (t), glycine-rich segments (g), polyalanine segments $(\alpha)$, mobile segments (m), $\beta$ crystallites ( $\beta$ ), nanofibrils (n). Based on Knight \& Vollrath (1999), Vollrath \& Knight (2001), Knight \& Vollrath (2001a,b) and Chen et al. (2001).



Figure 3. TEM of dragline silk from the orb web spider, Nephila edulis dispersed with a Silversen homogeniser at $4{ }^{\circ} \mathrm{C}$ and negatively stained in aqueous $2 \%$ ammonium molybdate. A fibril dispersed from a thread appears to be largely constructed from axially orientated nanofibrils with a diameter of ca. $3 \mathrm{~nm}$ lying approximately parallel. Scale bar, $100 \mathrm{~nm}$.

copolymer modelled on spider dragline silk ( $\mathrm{Qu}$ et al. 2000). (iv) Meridional small-angle X-ray scattering revealed two orders of a lamellar spacing of $8.4 \mathrm{~nm}$ (Yang et al. 1997). Calculations show that an extended glycinerich segment with an average of 30 residues, together with a polyalanine segment with an average of eight residues (figures derived from the spidroin I sequence published



Figure 4. As figure 1 but showing an area of nanofibrils dispersed to a greater extent. Scale bar, $100 \mathrm{~nm}$.

by Xu \& Lewis (1990)) can be accommodated into this lamellar spacing with a slight twisting or bending of the glycine-rich segments (C. Riekel, personal communication).

These observations strongly indicate that the spidroin nanofibril has the structure of an LLCE. For such a structure, variations in the detailed configuration of the glycinerich domains are likely to have only minor impact on the mechanical properties, provided that these segments remain mobile. By contrast, variations in the extent of $\beta$-crystallization are likely to have much larger effects. 
Thus it is hardly surprising that a comparative study of dragline silks suggests that the glycine-rich segments are highly variable but the polyalanine segments giving rise to the $\beta$-crystallites are highly conserved (Gatesy et al. 2001).

The mobility of the glycine-rich segments necessary for liquid crystalline elasticity has been demonstrated by ${ }^{13} \mathrm{C}$ and proton NMR (Jelinski 1998; Yang et al. 2000).

\section{(b) Liquid crystalline assembly}

A number of dragline silks from the most primitive spiders to the advanced orb web spinners have now been partially sequenced (Gatesy et al. 2001). This shows that their principal fibrous proteins, spidroins, like the analogous silkworm fibroins, are largely constructed from highly repetitive $\mathrm{AB}$ block copolymers showing a regular alternation of hydrophobic and less hydrophobic blocks. In spindroins, hydrophobic blocks consist of 4-10 alanine residues linked together, sometimes interrupted by a single serine residue. The less hydrophobic segments are longer and more variable in length and composition, and contain a high concentration of glycine residues.

Evidence that coulombic interactions play an important part in the assembly of spider dragline silk has been presented elsewhere (Chen et al. 2001).

Strong evidence that the dope precursor for orb web spider dragline silk is liquid crystalline has been reviewed elsewhere (Vollrath \& Knight 2001).

\section{(c) Elastomeric properties}

The bulk tensile properties of dragline silk from orb web spiders are those of an elastomer when wet (Gosline et al. 1984, 1994; Shao et al. 1999). The stress-strain curve is nonlinear (Gosline et al. 1984, 1986; Madsen et al. 1999), as it is in LCEs (Uchida 2000). The tensile behaviour of the nanofibril is less well characterized than the bulk properties of the thread. Two groups have attempted to determine X-ray moduli for spider dragline silk. Yang et al. (1997), using a loop formed from many fibres, showed that the axial period of $8.4 \mathrm{~nm}$ for dry Nephila silk stretched reversibly by $4 \%$ when the fibre was stretched by $10 \%$. Although this demonstrates short-range elasticity, it is not clear whether the greater bulk extension compared with the extension of the nanofibril arises from individual fibres or nanofibrils slipping past each other when strained. Similar objections together with uncertainties about the packing fraction apply to the work of Becker et al. (1994) who used a similar method to determine the $\mathrm{X}$-ray modulus. Future work with single fibres and a synchrotron X-ray source may provide detailed information about the tensile behaviour of the nanofibril, though it seems likely that the nanofibril will have a considerably higher modulus than the bulk material.

Although the terms LCE and LLCE have not so far been applied to spider dragline silk, this material has been modelled as an elastomeric network (Gosline et al. 1984; Termonia 1994) and many of these authors' conclusions are applicable to lamellar liquid crystalline nanofibrils as well as the amorphous matrix model they have used (see above).

\section{DISCUSSION}

We have presented strong evidence that the fibrous collagens and the dragline silk of orb web spiders are LLCEs.
Of the two routes by which LCEs can be assembled (see $\S 1$ ), nature appears to have mainly used the cross-linking of a monodomain liquid crystal rather than the more complex processing of an initially isotropic phase (see $\int 1$ ).

The suggested classification of the fibrous collagens and dragline silks as LLCEs has considerable predictive value. It may provide: (i) a testable hypothesis for the assembly of spider silk nanofibrils (see figure 2) that fits the currently known facts; (ii) new models for the origin of elasticity in collagens and silks; (iii) new approaches for the production of biomimetic materials; and (iv) the prediction that the exotic properties of LCEs may be found in biological elastomers.

\section{(a) Assembly of spider silk}

A hypothesis for the assembly of spider dragline silk as an LLCE is illustrated in figure 2. Spidroin is thought to be stored for use as compactly folded molecules (Hijirida et al. 1996). In these, the polyalanine segments are thought to assume an $\alpha$-helical conformation while the glycine-rich ones show a combination of $\beta$-turns and 'random coil' conformation (Hijirida et al. 1996). The flexible glycine-rich segments may allow the stiffer $\alpha$-helical segments to take up a nematic configuration within the compactly coiled molecule, as has been suggested by Pitard et al. (1997) for other proteins ((ii) in figure 2). The molecule may be rod-shaped and would be chiral, as are all proteins. The rod may be markedly amphiphilic, with one end more hydrophilic than the other. These characteristics would enable the molecules to assemble into nematic discotic units ((i) in figure 2$)$ in the very highly concentrated dope in the first and second limbs of the duct (Vollrath \& Knight 2001). The secretion of potassium and hydrogen ions into the lumen of the third limb of the duct (Chen et al. 2001; Vollrath \& Knight 2001) is thought to encourage the molecules to partly unfold by disrupting their water shell and altering coulombic forces (see (iii) in figure 2). They would then extend somewhat, align and be brought closer together in the extensional flow field of the draw-down taper found in the distal part of the duct (see (iv) in figure 2). This would align the molecules along the duct but with no preference for direction up or down it, resulting in an anti-parallel arrangement (see (iv) in figure 2). The retention of some hairpin loops arising from incomplete extension of the molecules would help to provide an anti-parallel arrangement. Incomplete rather than full extension is also probable on the grounds that it would reduce the force required to form the silk thread. Hydrophobic effects operating on the repetitive amphiphilic $A B$ block copolymeric structure would enable staggered, antiparallel chains to assemble in a lamellar structure. In addition, coulombic forces arising from charged amino acids may play a role in the formation of the lamellar nanofibrils (Chen et al. 2001), as in collagens. As the hydrophobic polyalanine segments of spidroin align and are drawn closer together by extensional flow they would find themselves in an increasingly hydrophobic environment and this could trigger their conversion from an $\alpha$-helical to a $\beta$-pleated structure resulting in the formation of numerous interchain hydrogen bonds (see (v) in figure 2). The latter would act as multifunctional crosslinks at nodes between the more mobile glycine-rich segments (see (v) in figure 2). Thus the assembly of the 
thread can be seen as a liquid-crystalline phase transition involving separation into polymer-rich and solvent-rich phases (Knight et al. 2000).

\section{(b) Tensile properties}

It may be possible to apply LCE theory to silks and collagens to understand their tensile properties further and, in particular, to predict the influence of variation in the length of mobile segments and cross-link density in different silks. Although extensible molecules lying between, and tightly bonded to, rather stiff nanofibrils constructed from spidroin I may be important for the extensibility of spider silk, this could be incorporated into an LCE model. An extended Gaussian theory may be applicable to biological LCEs, but this mechanism may not be solely responsible for their elastomeric force as energy storage in helical springs, such as $\beta$-turns or spirals, and other entropic effects may also be involved (Tatham \& Shewry 2000). The complexity of the mechanical behaviour of a biological LCE may be further increased because liquid crystals are highly tunable (Collings 1990). Thus, changes in the content of water, ions and small molecules may account for some of the variation in the properties of spider dragline silk from day-to-day, individual-to-individual and species-to-species observed by Madsen et al. (1999). It may also help to account for the variation in the structure and mechanical behaviour of fibrillar collagens in health and disease (Royce \& Steinman 1993) and with collagen type (Ricard-Blum et al. 2000).

\section{(c) Production of biomimetic materials}

It may be possible to use LCE theory to design polymers that mimic the properties of fibrous collagens, dragline silks and other biological elastomers. Three routes might be used for this: (i) genetic engineering of spidroin analogues, (ii) direct use of natural amphiphilic block copolymeric proteins from agricultural products such as wheat germ (Tatham \& Shewry 2000), and (iii) conventional synthesis of block copolymers. The ability of amphiphilic block copolymers to unfold and form lamellar liquid crystals, sometimes with nanofibrillar textures (Bauer et al. 1995; Morishima 2000; Roschinski \& Kulicke 2000) is particularly important in this connection and provides a possible route for the production of nanocomposite materials.

\section{(d) Exotic properties}

LCEs have a range of exotic properties (Texeira 1999), some of which may have been exploited by living organisms. These may include: transparency (Anderson \& Ström 1991), as in the collagenous cornea; piezoelectricity (Meier \& Finkelmann 1993), which may provide a feedback loop relating mechanical loading to increased synthetic activity in connective tissues; and anomalous sensitivity of shape change to electrical fields and mechanical strain (Verwey \& Warner 1995), which may help to define collagen orientations in morphogenesis and tissue remodelling.

Finally, we note that other nano- or microfibrils, including those of elastin (Kim et al. 1999), fibrillin (Keene et al. 1991), fibrin (Mosesson et al. 1995), Bombyx mori silk (Putthanarat et al. 2000) and ciliary rootlets (Anstrom 1992) may also be LLCEs. The transversely banded costas of protists (Brugerolle 1999) may be of particular interest for the development of biomimetic microactuators as they show a remarkable phase transition, reversibly shortening on the addition of low concentrations of calcium ions.

We thank David Porter for encouraging us to think of collagen and spider silk as LCEs; the Danish Science Research Council (SNF), the Science Faculty of Aarhus University, the Carlsberg Foundation, the Danish Academy of Science, the British Biological and Engineering Research Councils (BBSRC, EPSRC) and the European Science Foundation (ESF) for funding; Terry Chen, Peter Fratzl, David Kaplan, Fred Keeley, Arthur Tatham and Carlo Knupp, Eugene Terentjev and John Gosline for helpful discussion; and Academic Press for permission to reproduce figure 1. D.P.K. thanks the Biological Imaging Centre, Southampton University for technical assistance.

\section{REFERENCES}

Anderson, D. M. \& Ström, P. 1991 Polymerized lyotropic liquid crystals as contact lens materials. Physica A 176, 151-167.

Anstrom, J. A. 1992 Organization of the ciliary basal apparatus in embryonic cells of the sea urchin Lytechinus pictus. Cell Tiss. Res. 269, 305-313.

Barclay, G. G. \& Ober, C. K. 1993 Liquid-crystalline and rigirod networks. Prog. Polym. Sci. 18, 899-945.

Bauer, H., Böse, N. \& Stern, P. 1995 Viscoplastic flow and shear thickening in concentrated diblock copolymer solutions. Colloid Polym. Sci. 273, 480-489.

Becker, M. A., Mahoney, D. V., Lenhert, P. G., Eby, R. K., Kaplan, D. \& Adams, W. W. 1994 X-ray moduli of silk fibres from Nephila clavipes and Bombyx mori. Am. Chem. Soc. Symp. Ser. 544, 185-195.

Bouligand, Y., Cladis, P. E., Liebert, L. \& Strzelecki, L. 1974 Study of thin films of polymerized liquid crystals. Mol. Crystall. Liq. Cryst. 25, 233.

Brown, E. M., King, G. \& Chen, J. M. 1997 Model of the helical portion of a type I collagen microfibril. F. Am. Leather Chem. Assoc. 92, 1-7.

Brugerolle, G. 1999 Fine structure of Pseudotrypanosoma giganteum of Porotermes, a trichomonad with a contractile costa. Eur. F. Protistol. 35, 121-128.

Chan, Y. N. C., Craig, G. S. W., Schrock, R. R. \& Cohen, R. E. 1992 Synthesis of palladium and platinum nanoclusters within microphase-separated diblock copolymers. Chem. Mater. 4, 885-894.

Chapman, J. A., Tzaphlidou, M., Meek, K. M. \& Kadler, K. E. 1990 The collagen fibril—a model system for studying the staining and fixation of a protein. Electron Microsc. Rev. 3, 143-182.

Chen, X., Knight, D. P. \& Vollrath, F. 2001 Rheological characterization of Nephila spidroin solution. Int. F. Am. Chem. Soc. (Submitted.)

Collings, P. J. 1990 Liquid crystals: nature's delicate phase of matter, 1st edn. Princeton University Press.

de Gennes, P. G. 1969 Possibilités offertes par la réticulation de polymères en présence d'un cristal liquide. Phys. Lett. A. 28, 725 .

de Gennes, P.G. 1974 The physics of liquid crystals, 1st edn. Oxford: Clarendon Press.

de Gennes, P. G. 1975 Rèflexions sur un type de polyméres nématiques. Crit. Rev. Acad. Sci. B 281, 101-103.

Fischer, P., Schmidt, C. \& Finkelmann, H. 1995 Amphiphilic liquid crystalline networks. Phase-behaviour and alignment by mechanical fields. Macromol. Rap. Commun. 16, 435-447.

Fraaije, J. G. E. M., Zvelindovsky, A. V., Sevink, G. J. A. \& 
Maurits, N. M. 2000 Modulated self-organization in complex amphiphilic systems. Mol. Simul. 25, 131-144.

Fraser, R. D. B., MacRae, T. P. \& Miller, A. 1987 Molecular packing in type I collagen fibrils. F. Mol. Biol. 193, 115-126.

Fraser, W. D. 1998 The collagen crosslinks pyridinoline and deoxypyridinoline: a review of their biochemistry, physiology, measurement, and clinical applications. Clin. Ligand Assay 21, 102-110.

Gatesy, J., Hayashi, C., Motriuk, D., Woods, J. \& Lewis, R. 2001 Extreme diversity, conservation and convergence of spider silk fibroin sequences. Science 291, 2603-2605.

Gathercole, L. J., Miles, M. J., McMaster, T. J. \& Holmes, D. F. 1993 Scanning probe microscopy of collagen-I and Pn-collagen-I assemblies and the relevance to scanningtunneling-microscopy contrast generation in proteins. $\mathcal{F}$. Chem. Soc. Farad. Trans. 89, 2589-2594.

Gosline, J., Denny, M. \& DeMont, M. 1984 Spider silk as rubber. Nature Lond. 309, 551-552.

Gosline, J. M., DeMont, M. E. \& Denny, M. W. 1986 The structure and properties of spider silk. Endeavour N. S. 10, 31-43.

Gosline, J. M., Pollack, C. C., Guerette, P. A., Cheng, A., DeMont, M. E., Denny, M. W. 1994 Elastomeric network models for the frame and viscid silks from the orb web of the spider Araneus diadematus. In Silk polymers. Materials science and biotechnology. (ed. D. Kaplan, W. W. Adams, B. Farmer \& C. Viney), pp. 328-341. Washington DC: American Chemical Society.

Grubb, D. T. \& Ji, G. 1999 Molecular chain orientation in supercontracted and re-extended spider silk. Int. f. Biol. Macromol. 24, 203-210.

Hamley, I. W. 2000 The effect of shear on ordered block copolymer solutions. Curr. Opin. Colloid Int. Sci. 5, 342-350.

Hepworth, D. G., Gathercole, L. J., Knight, D. P., Feng, D. \& Vincent, J. F. V. 1994 Correlation of ultrastructure and tensile properties of a collagenous composite material, the egg capsule of the dogfish, Scyliorhinus spp. a sophisticated collagenous material. F. Struct. Biol. 112, 231-240.

Hijirida, D. H., Do, K. G., Michal, C., Wong, S., Zax, D. \& Jelinski, L. W. 1996 C13 NMR of Nephila clavipes major ampullate silk gland. Biophys. F. 71, 3442-3447.

Hu, X. W., Knight, D. P. \& Chapman, J. A. 1997 The effect of non-polar liquids and non-ionic detergents on the ultrastructure and assembly of rat tail tendon collagen fibrils in vitro. Biochim. Biophys. Acta Gen. Subj. 1334, 327-337.

Hukins, D. W. L., Woodhead-Galloway, J. \& Knight, D. P. 1976 Molecular tilting in dried elastoidin and its implications for the structures of other collagen fibrils. Biochem. Biophys. Res. Commun. 73, 1049-1055.

Hukins, D. W. L. \& Woodhead-Galloway, J. 1977 Collagen fibrils as examples of smectic A biological fibres. Mol. Crystall. Liq. Cryst. 41, 33-39.

Hukins, D. W. L. \& Woodhead-Galloway, J. 1978 Liquid crystalline model for the organization of molecules in collagen fibrils. Biochem. Soc. Trans. 6, 238-239.

Hulmes, D. J. S., Miller, A., Parry, D. A. D., Piez, K. A. \& Woodhead-Galloway, J. 1973 Analysis of the primary structure of collagen for the origins of molecular packing. F. Mol. Biol. 79, 137-148.

Hulmes, D. J. S., Weiss, T. J., Prockop, D. J. \& Fratzl, P. 1995 Radial packing, order, and disorder in collagen fibrils. Biophys. F. 68, 1661-1670.

Jelinski, L. W. 1998 Establishing the relationship between structure and mechanical function in silks. Curr. Opin. Solid State Mater. Sci. 3, 235-237.

Keene, D. R., Maddox, B. K., Kuo, H. J., Sakai, L. Y. \& Glanville, R. W. 1991 Extraction of extendable beaded structures and their identification as fibrillin-containing extracellular matrix microfibrils. F. Histochem. Cytochem. 39, 441-449.
Kim, J. S., McHugh, S. K. \& Swager, T. M. 1999 Nanoscale fibrils and grids; aggregated structures from rigid-rod conjugated polymers. Macromolecules 32, 1500-1507.

King, M. R., White, S. A., Smith, S. D. \& Spontak, R. J. 1999 Mesogel networks via selective swelling of lamellar triblock copolymers. Langmuir 5, 7886-7889.

Knight, D. P. \& Hunt, S. 1974 Fibril structure of collagen in egg capsule of dogfish. Nature Lond. 249, 379-380.

Knight, D. P. \& Vollrath, F. 1999 Liquid crystals and flow elongation in a spider's silk production line. Proc. $R$. Soc. Lond. B 266, 519-523. (DOI 10.1098/rspb.1999.0667.)

Knight, D. P. \& Vollrath, F. 2001 a A comparison of liquid crystal spinning of selachian egg case ply sheet and orb web spider dragline filaments. Macromolecules 2, 323-324.

Knight, D. P. \& Vollrath, F. $2001 b$ Changes in element composition in the secretory pathway for dragline silk in a Nephila spider. Naturwissenschaften 88, 179-182.

Knight, D. P., Feng, D., Stewart, M. \& King, E. 1993 Changes in macromolecular organisation in collagen assemblies during secretion in the nidamental gland and formation of the egg capsule wall in the dogfish Scyliorhinus canicula. Phil. Trans. R. Soc. Lond. B 341, 419-436.

Knight, D. P., Feng, D. \& Stewart, M. 1996 Structure and function of the selachian egg case. Biol. Rev. 76, 81-111.

Knight, D. P., Knight, M. M. \& Vollrath, F. 2000 Beta transition and stress-induced phase separation in the spinning of spider dragline silk. Int. F. Biol. Macromol. 27, 205-210.

Knott, L. \& Bailey, A. J. 1998 Collagen cross-links in mineralizing tissues: a review of their chemistry, function, and clinical relevance. Bone 22, 181-187.

Knupp, C. \& Squire, J. 1998 X-ray diffraction analysis of the 3D organization of collagen fibrils in the wall of the dogfish egg case. Proc. R. Soc. Lond. B 265, 2177-2186. (DOI 10.1098/rspb.1998.0556.)

Knupp, C. \& Squire, J. 2001 A new twist in the collagen story-the type VI segmented supercoil. EMBO f. 20, 372-376.

Knupp, C., Chew, M. \& Squire, J. 1998 Collagen packing in the dogfish egg case wall. F. Struct. Biol. 122, 101-110.

Kümmerlen, J., Van Beek, J., Vollrath, F. \& Meier, B. 1996 Local structure in spider dragline silk investigated by twodimensional spin-diffusion nuclear magnetic resonance. Macromolecules 29, 2920-2928.

Lees, S. 1998 Interpreting the equatiorial diffraction pattern of collagenous tissues in the light of molecular motion. Biophys. F. 75, 1058-1061.

Legge, C. H., Davis, F. J. \& Mitchell, G. R. 1991 Memory effects in liquid-crystal elastomers. F. Phys. II France 1, 1253-1261.

Leikin, S., Rau, D. C. \& Parsegian, V. A. 1995 Temperaturefavored assembly of collagen is driven by hydrophilic not hydrophobic interactions. Nature Struct. Biol. 2, 205-210.

Mao, Y., Warner, M., Terentjev, E. M. \& Ball, R. C. 1998 Finite extensibility effects in nematic elastomers. F. Chem. Phys. 108, 8743-8748.

Madsen, B., Shao, Z. \& Vollrath, F. 1999 Variability in the mechanical properties of spider silks on three levels: interspecific, intraspecific and intraindividual. Int. F. Biol. Macromol. 24, 301-306.

Martin, R., Farjanel, J., Eichenberger, D., Colige, A., Kessler, E., Hulmes, D. J. S. \& Giraud-Guille, M.-M. 2000 Liquid crystalline ordering of procollagen as a determinant of threedemensional extracellular matrix architecture. f. Mol. Biol. 301, 11-17.

Meier, W. \& Finkelmann, H. 1993 Piezoelectricity of cholesteric elastomers. 1 . Influence of the helicoidal pitch on the piezoelectric coefficient. Macromolecules 26, 1811-1817.

Misof, K., Rapp, G. \& Fratzl, P. 1997 A new molecular model 
for collagen elasticity based on synchtrotron X-ray scattering evidence. Biophys. F. 72, 1376-1381.

Mitchell, G. R., Coulter, M., Davis, F. J. \& Guo, W. 1992 The effect of the spacer length on the nature of coupling in side-chain liquid crystal polymers and elastomers. F. Phys. 12, 1121-1132.

Morishima, Y. 2000 Self assembling amphiphilic polyelectrolytes and their nanostructure. Chin. F. Polym. Sci. 18, 323-324.

Mosesson, M. W., Siebenlist, K. R. \& Hanfield, J. F. 1995 The covalent structure of factor XIIIA cross-linked fibrinogen fibrils. F. Struct. Biol. 115, 88-101.

Northolt, M. G. \& Sikkema, D. J. 1991 Lyotropic main chain liquid-crystal polymers. Adv. Polym. Sci. 98, 115-117.

Ortolani, F., Raspanti, M. \& Marchini, M. 1994 Correlations between amino acid hydrophobicity scales and stain exclusion capacity of type I native collagen fibrils. F. Electron Microsc. 43, 32-38.

Parry, D. A. D. 1988 The molecular and fibrillar structure of collagen and its relationship to the mechanical properties of connective tissue. Biophys. Chem. 29, 195-209.

Pitard, E., Garel, T. \& Orland, H. 1997 Protein folding, anisotropic collapse and blue phases. F. Phys. I 17, 1201-1210.

Putthanarat, S., Stribeck, N., Fossey, S. A., Eby, R. K. \& Adams, W. W. 2000 Investigation of the nanofibrils of silk fibers. Polymer 41, 7735-7747.

Qu, Y., Payne, S. C., Apkarian, R. P. \& Conticello, V. P. 2000 Self-assembly of a polypeptide multi-block copolymer modelled on dragline silk protein. F. Am Chem. Soc. 122, 5014-5015.

Ricard-Blum, S., Dublet, B. \& Van der Rest, M. 2000 Unconventional collagens. New York: Oxford University Press.

Riekel, C., Bränden, C., Craig, C., Ferrero, C., Heidelbach, G. \& Müller, M. 1999 Aspects of X-ray diffraction on single spider fibers. Int. F. Biol. Macromol. 24, 179-186.

Roschinski, C. \& Kulicke, W.-M. 2000 Rheological characterization of aqueous hydroxypropyl cellulose solutions close to phase separation. Macromol. Chem. Phys. 201, 2031-2040.

Rosenblatt, J., Devereux, B. \& Wallace, D. G. 1993 Dynamic rheological studies of hydrophobic interactions in injectable collagen biomaterials. F. Appl. Polym. Sci. 50, 953-963.

Royce, P. M. \& Stenimann, B. (eds) 1993 Connective tissue and its heritable disorders. New York: Wiley-Liss.

Schneider, A., Muller, S. \& Finkelmann, H. 2000 Lyotropic mesomorphism of AB block copolymers in nematic solvents. Macromol. Chem. Phys. 201, 184-191.

Shao, Z., Young, R. J. \& Vollrath, F. 1999 The effects of solvents on spider silk studied by mechanical testing and single-fibre Raman spectroscopy. Int. F. Biol. Macromol. 24, 295-300.

Simmons, A., Michal, C. \& Jelinski, L. 1996 Molecular orientation and two-component nature of the crystalline fraction of spider dragline silk. Science 271, 84-87.
Sirichaisit, S., Young, R. J. \& Vollrath, F. 1999 Molecular deformation in spider dragline silk subjected to stress. Polymer 41, 1223-1227.

Tatham, A. S. \& Shewry, P. R. 2000 Elastomeric proteins: biological roles, structures and mechanisms. TIBS 25, 567-571.

Termonia, Y. 1994 Molecular modeling of spider silk elasticity. Macromolecules 27, 7378-7381.

Texeira, P. I. C. 1999 Dynamics of reorientation of a constrained nematic elastomer. Eur. Phys. F. B 9, 471-477.

Texeira, P. I. C. \& Warner, M. 1999 Dynamics of soft and semisoft nematic elastomers. Phys. Rev. E 60, 603-609.

Treloar, L. R. G. 1975 The physics of rubber elasticity. Oxford: Clarendon Press.

Uchida, N. 2000 Soft and nonsoft structural transitions in disordered nematic networks. Phys. Rev. E 62, 5119-5134.

Van Beek, J. D., Kümmerlen, D., Vollrath, F. \& Meier, B. H. 1999 Supercontracted spider dragline silk: a solid-sate NMR study of the local structure. Int. F. Biol. Macromol. 24, 173-178.

Verwey, G. C. \& Warner, M. 1995 Soft rubber elasticity. Macromolecules 28, 4303-4306.

Vincent, J. F. V. 1982 Structural materials. London: Macmillan.

Vinee, P., Meurer, B., Constantinesco, A., Kohlberger, B., Hauensterin, K. H., Lauberberger, J. \& Petkov, S. 1993 Characterization of human aortic collagen's elasticity by nuclear magnetic resonance. Magn. Reson. Imag. 11, 395399.

Vollrath, F. \& Knight, D. P. 2001 Liquid crystalline spinning in nature. Nature Lond. 410, 541-548.

Wallace, D. 1985 The role of hydrophobic bonding in collagen fibril formation-a quantitative model. Biopolymers 24, 1705-1720.

Warner, M. \& Terentjev, E. M. 1996 Nematic elatomers-a new state of matter? Prog. Polym. Sci. 21, 853-891.

Warner, M., Gelling, K. P. \& Vilgis, T. A. 1988 Theory of nematic networks. f. Chem. Phys. 88, 4008-4013.

Woodhead-Galloway, J. \& Knight, D. P. 1977 Some observations on the fine structure of elastoidin. Proc. R. Soc. Lond. B 195, 355-364.

Xu, M. \& Lewis, R. V. 1990 Structure of a protein superfiber: spider dragline silk. Proc. Natl Acad. Sci. USA 87, 71207124.

Yang, Z., Grubb, D. T. \& Jelinski, L. W. 1997 Small angle X-ray scattering of spider dragline silk. Maromolecules 30, 8254-8261.

Yang, Z. T., Liivak, O., Seidel, A., LaVerde, G., Zax, D. B. \& Jelinski, L. W. 2000 Supercontraction and backbone dynamics in spider silk: C-13 and H-2 NMR studies. F. Am. Chem. Soc. 122, 9019-9025.

Yeh, W.-Y. \& Young, R. J. 1999 Molecular deformation processes in aromatic high modulus polymer fibres. Polymer 40, 857-870. 